\title{
Replacement of Dopaminergic Medication with Subthalamic Nucleus Stimulation in Parkinson's Disease: Long-Term Observation
}

\author{
Luigi M. Romito, MD, PhD, ${ }^{1,2}$ Maria Fiorella Contarino, $\mathrm{MD},{ }^{3}$ Nicola Vanacore, $\mathrm{MD},{ }^{4}$ \\ Anna Rita Bentivoglio, MD, PhD, ${ }^{3}$ Massimo Scerrati, MD, ${ }^{5}$ and Alberto Albanese, $\mathrm{MD}^{1,2 *}$ \\ ${ }^{1}$ Fondazione IRCCS Istituto Neurologico “Carlo Besta," Milano, Italy \\ ${ }^{2}$ Università Cattolica del Sacro Cuore, Milano, Italy \\ ${ }^{3}$ Istituto di Neurologia, Università Cattolica del Sacro Cuore, Roma, Italia \\ ${ }^{4}$ Centro Nazionale di Epidemiologia, Istituto Superiore di Sanita, Roma, Italia \\ ${ }^{5}$ Clinica di Neurochirurgia, Università degli Studi, Ancona, Italy
}

\begin{abstract}
Stimulation of the subthalamic nucleus (STN) is an effective treatment for advanced Parkinson's disease (PD), but the medication requirements after implant are poorly known. We performed a long-term prospective evaluation of 20 patients maintained at stable dopaminergic therapy for 5 years after bilateral STN implants, who were evaluated 6 months, 1 year, 3 years, and 5 years after surgery. We measured, during the entire observation period, the effect of deep brain stimulation on motor and functional outcome measures, the levodopa equivalent daily dose and the total electrical energy delivered. At 5 years, the UPDRS motor score had improved by $54.2 \%$ and levodopa equivalent dose was reduced by $61.9 \%$, compared with preimplant. Dopaminergic medication remained stable during the observation period,
\end{abstract}

but energy was progressively increased over time. Rest tremor, rigidity, gait, lower and upper limb akinesia, and total axial score were improved in decreasing order. Postural stability and speech improved transiently, whereas on-period freezing of gait, motor fluctuations and dyskinesias recovered durably. Functional measures did not show improvement in autonomy and daily living activities after STN implant. Chronic STN stimulation allows to replace for dopaminergic medications in the long-term at the expense of an increase of the total energy delivered. This is associated with marked improvement of motor features without a matching benefit in functional measures. (C) 2008 Movement Disorder Society

Key words: Parkinson's disease; surgery; deep brain stimulation; subthalamic nucleus; levodopa; dopamine agonists
Parkinson's disease (PD) is a progressive disorder affecting the motor system. Currently available medications, although providing optimal symptomatic benefit, often lead to troublesome side-effects, like motor fluctuations and on-period dyskinesias, especially in the long term. High frequency stimulation of the subthalamic nucleus (STN) has been effectively used to treat PD patients, providing significant symptomatic

*Correspondence to: Alberto Albanese, Fondazione IRCCS, Istituto Neurologico "Carlo Besta", Via G. Celoria 11, I-20133, Milano, Italy. E-mail: alberto.albanese@unicatt.it

Potential conflict of interest: None reported.

Received 3 April 2008; Revised 14 October 2008; Accepted 19 October 2008

Published online 18 December 2008 in Wiley InterScience (www. interscience.wiley.com). DOI: 10.1002/mds.22390 relief and allowing to reduce antiparkinsonian medication. ${ }^{1,2}$ The magnitude of motor score improvement after STN stimulation exhibits a dose-response relationship with preoperative response to levodopa challenge, ${ }^{3}$ but the extent to which STN stimulation can replace medication in the long term is poorly known. One study reported that levodopa equivalents (LEDD) was reduced by $25 \%$ after 5 years, ${ }^{4}$ whereas another study indicated a $57 \%$ decrease. ${ }^{5}$ Different strategies to reduce dopaminergic treatment were used in these studies, which did not report the amount of total electrical energy delivered (TEED) through the electrodes. We performed a prospective study aimed to reduce and stabilize dopaminergic medication after STN stimulation and report on the long-term clinical outcome and the TEED. 


\section{PATIENTS AND METHODS}

We studied the first 20 (11 men and 9 women) consecutive patients who received STN implants and reached the fifth year of follow-up. All the patients received a diagnosis of PD according to the UK Parkinson's Disease Brain Bank criteria. ${ }^{6}$ They all had excellent and sustained response to levodopa, with motor complications, such as disabling motor fluctuations with prolonged and at least occasionally unpredictable "off" periods (patients spent at least $25 \%$ of the waking day in the off state) and on-period dyskinesias; were HoehnYahr stage $\geq \mathrm{III}$ in the practically defined off condition $^{7}$; fulfilled recognized inclusion and exclusion criteria (heart pacemaker bearer, mild parkinsonian features or unstable drug regimen, cognitive impairment, ongoing psychiatric problems, prior brain surgery or inability to comply with the study protocol $)^{2}$ and the recommendations of the CAPSIT-PD panel. ${ }^{7}$ The occurrence of mild, dopaminergic medication-dependent, hallucinations were not considered a reason for exclusion, if these mild symptoms could be fully controlled by low doses of clozapine (up to 25-mg daily).

The study protocol was approved by the hospital internal review board. The eligible patients signed an informed consent before entering the study; they were all evaluated, implanted and followed-up at the same institution.

Bilateral simultaneous STN implants were performed in all patients using a standard stereotactic technique. ${ }^{2}$ The patients had a mean $( \pm$ SD) age at implant of 56.4 $( \pm 6.9)$ years (disease duration: $14.3 \pm 6.2$ ). Dopamine agonists were withdrawn 1 week before surgery and levodopa the evening before. Intraoperative test stimulation (pulses of $60 \mu \mathrm{S}$ at a frequency of $130 \mathrm{~Hz}$ ) was performed by two neurologists while the patient was awake, before implanting the permanent stimulating electrode. Approximately 1 week later, two Itrel II or one Kinetra (Medtronic, Minneapolis, MN) implantable pulse generators (IPG) were placed in the subclavear region. Stimulation parameters were then checked without medication to achieve optimal control of motor symptoms and to identify the threshold for side effects. The same procedure was repeated 1 and 3 months after implant and at all scheduled follow-up evaluations; aim of the procedure was to identify the stimulation settings and choice of stimulating contacts providing the best possible control on motor signs. Medication was gradually reintroduced after implant, and was maintained at each followup evaluation to the minimum dose necessary to permit optimal motor control in addition to stimulation.

The patients were evaluated preoperatively and then $6,12,18$ months and 2, 3, 5 years after the implant.
Preoperative evaluations were performed in the morning, in the practically defined off condition ${ }^{7}$ and in the best on condition following the first morning dose of levodopa. Each postoperative test session was performed in the morning and consisted of three consecutive UPDRS evaluations: (a) in the practically defined off condition without antiparkinsonian medication and with stimulation turned off; (b) without antiparkinsonian medication, 30 min after switching stimulation on; and (c) with antiparkinsonian medication and stimulation. Condition (a) intended to evaluate the progression of PD motor signs without the influence of treatment; condition (b) was used to measure the efficacy of STN stimulation alone on PD motor signs; and condition (c) served to evaluate the patients' functioning during their best motor condition.

Upper limb akinesia was defined as the sum of the following UPDRS motor items: finger and hand tapping (Items 23 and 24), hand pronation-supination (Item 25). Lower limb akinesia was determined using the UPDRS foot tapping subscore (Item 26). The total axial score encompassed the following UPDRS motor items: speech (Item 18), gait (Item 29), and postural stability (Item 30). Freezing of gait was evaluated using Item 14 of the UPDRS part II. The duration of off-periods was determined based on Item 39 of the UPDRS, part IV. Dyskinesias were calculated by the sum of the dyskinesia duration and disability UPDRS part IV scores (Items 32 and 33); variations of dyskinesias were measured by comparing the postoperative condition with medication and stimulation turned on to the preoperative state with medication. Activities of daily living were evaluated with the UPDRS part II score and the Schwab and England (S\&E) functional scale in the patients' best functional state (condition $c$ above). LEDD (measured in milligrams) was obtained by adding to the standard levodopa dose all other dopaminergic medications converted to the relative potency of standard levodopa; TEED (measured in $\mu \mathrm{J}$ ) was computed by the formula TEED $=\left(\right.$ amplitude $^{2} \times$ pulse width $\times$ frequency rate)/impedance. ${ }^{8}$ Adverse events were classified as transient, persistent (if not improved by turning off stimulation for a short time), stimulation-induced (present at optimal stimulation parameters, but improved when stimulation was turned off or stimulation parameters were modified), devicerelated, or unrelated to the procedure or stimulation. ${ }^{9}$

Repeated measures of analysis of variance were applied to evaluate differences in clinical findings during follow-up. Bonferroni's correction for comparisons between different times was then applied. The chisquare test with Fisher's correction was applied to 
TABLE 1. Efficacy of stimulation and progression of motor signs evaluated at different times after STN implant ( \pm SD)

\begin{tabular}{|c|c|c|c|c|c|}
\hline & Baseline & $6 \mathrm{mo}$ & $1 \mathrm{yr}$ & $3 \mathrm{yr}$ & $5 \mathrm{yr}$ \\
\hline \multicolumn{6}{|c|}{ Efficacy of STN stimulation on motor features ${ }^{\mathrm{a}}$} \\
\hline UPDRS-motor score & $60.5 \pm 9.5$ & $29.5 \pm 10.3^{* * *}$ & $30.1 \pm 13.4 * * *$ & $31.6 \pm 12.1^{* * *}$ & $27.7 \pm 12.6^{* * *}$ \\
\hline Rest tremor & $8.4 \pm 5.9$ & $1.8 \pm 2.3 * * *$ & $1.3 \pm 3.2 * * *$ & $1.1 \pm 1.8^{* * *}$ & $0.4 \pm 1.0^{* * *}$ \\
\hline Rigidity & $11.2 \pm 3.7$ & $6.4 \pm 3.4 * * *$ & $6.4 \pm 3.7 * * *$ & $6.6 \pm 4.2 * * *$ & $4.9 \pm 3.8 * * *$ \\
\hline Upper limb akinesia & $13.8 \pm 4.3$ & $9.2 \pm 5.2 * * *$ & $9.0 \pm 5.9 * *$ & $9.5 \pm 4.7 * * *$ & $8.3 \pm 5.2 * * *$ \\
\hline Lower limb akinesia & $5.3 \pm 2.2$ & $2.9 \pm 2.1 * * *$ & $3.1 \pm 2.7 * *$ & $3.2 \pm 2.1 * *$ & $3.0 \pm 2.1 * * *$ \\
\hline Total axial score & $6.4 \pm 2.0$ & $3.0 \pm 2.0 * * *$ & $3.1 \pm 1.9 * * *$ & $4.0 \pm 1.8 * *$ & $4.1 \pm 1.7^{* * *}$ \\
\hline Speech & $2.3 \pm 0.6$ & $1.4 \pm 0.8^{* *}$ & $1.7 \pm 0.9$ & $1.8 \pm 0.8$ & $2.0 \pm 1.0$ \\
\hline Postural stability & $1.8 \pm 1.2$ & $0.7 \pm 0.9 * * *$ & $0.7 \pm 0.8 * * *$ & $0.9 \pm 0.8$ & $1.0 \pm 0.8$ \\
\hline Gait & $2.4 \pm 1.0$ & $0.9 \pm 0.8 * * *$ & $0.8 \pm 0.8^{* * *}$ & $1.3 \pm 0.9 * * *$ & $1.2 \pm 0.8 * * *$ \\
\hline Dyskinesia score & $4.1 \pm 1.7$ & $0.4 \pm 0.8 * * *$ & $0.6 \pm 0.9 * * *$ & $0.7 \pm 1.1 * * *$ & $0.8 \pm 1.5^{* * *}$ \\
\hline Off-period duration & $2.0 \pm 0.8$ & $0.0 \pm 0.2 * * *$ & $0.1 \pm 0.3 * * *$ & $0.1 \pm 0.3 * * *$ & $0.0 \pm 0.2 * * *$ \\
\hline \multicolumn{6}{|c|}{ Efficacy of STN stimulation on functional measures ${ }^{b}$} \\
\hline UPDRS Activities of daily living & $10.9 \pm 8.4$ & $7.4 \pm 5.6$ & $7.5 \pm 5.5$ & $8.9 \pm 6.6$ & $8.6 \pm 5.8$ \\
\hline Freezing of gait & $2.4 \pm 0.6$ & $0.6 \pm 0.7 * * *$ & $0.7 \pm 0.6 * * *$ & $0.8 \pm 0.7 * *$ & $1.0 \pm 0.8^{* *}$ \\
\hline Schwab \& England Scale & $76.8 \pm 15.6$ & $90.8 \pm 7.1 * *$ & $92.4 \pm 7.1^{*}$ & $85.8 \pm 11.7$ & $84.7 \pm 13.1$ \\
\hline \multicolumn{6}{|c|}{ Evaluation of underlying PD after STN stimulation ${ }^{\mathrm{c}}$} \\
\hline UPDRS-motor score & $60.5 \pm 9.5$ & $57.7 \pm 16.2$ & $56.4 \pm 14.5$ & $56.1 \pm 16.9$ & $55.8 \pm 17.8$ \\
\hline \multicolumn{6}{|l|}{ Axial scores } \\
\hline Speech & $2.3 \pm 0.6$ & $1.9 \pm 0.9$ & $2.2 \pm 1.0$ & $2.2 \pm 1.2$ & $2.2 \pm 1.0$ \\
\hline Rigidity & $11.2 \pm 3.7$ & $11.4 \pm 4.3$ & $10.7 \pm 3.8$ & $11.7 \pm 4.4$ & $11.4 \pm 4.7$ \\
\hline Postural stability & $1.8 \pm 1.2$ & $1.1 \pm 0.7$ & $1.0 \pm 0.8$ & $1.6 \pm 1.2$ & $1.5 \pm 1.3$ \\
\hline Gait & $2.4 \pm 1.0$ & $1.9 \pm 1.0^{*}$ & $1.8 \pm 0.8$ & $2.0 \pm 1.2$ & $2.0 \pm 1.2$ \\
\hline
\end{tabular}

All the 20 patients are included in each evaluation.

${ }^{a}$ Evaluated without antiparkinsonian medication and with stimulation turned on.

${ }^{\mathrm{b} E v a l u a t e d ~ i n ~ t h e ~ p a t i e n t s ' ~ b e s t ~ f u n c t i o n a l ~ s t a t e, ~ w i t h ~ c u r r e n t ~ a n t i p a r k i n s o n i a n ~ m e d i c a t i o n ~ a n d ~ w i t h ~ s t i m u l a t i o n ~ t u r n e d ~ o n . ~}$

${ }^{\mathrm{c}}$ Evaluated with stimulation turned off and without antiparkinsonian medication.

$* P<0.05, * * P<0.005, * * * P<0.0005$ compared with baseline values. All the other comparisons were not significant.

determine differences in the frequencies of categorical variables. The mean values of clinical variables were compared by means of the Student's t-test (unpaired and paired data) and the Wilcoxon signed-rank test. Any $P$ values $<0.05$ were considered statistically significant. All values were expressed as means \pm SD. Statistical analysis was performed with SPSS software (http://www.spss.com/spss/, release 12.0).

\section{RESULTS}

At baseline, the mean motor improvement with levodopa was $58.4 \%$ (range: 38.2-82.1); the mean HoehnYahr stage was $4.4 \pm 0.9$ in the off-state and $2.6 \pm$ 0.8 during the on-state. Comparison of the UPDRS motor score 5 years after implant with stimulation on, but without medication, to the preoperative condition without medication showed a $54.2 \%$ improvement (Table 1). This was not evenly distributed: rest tremor had the most remarkable changes, followed by rigidity, gait, lower limb akinesia, upper limb akinesia, and total axial score. There was no deterioration during the observation period. By contrast, postural stability markedly improved during the first year after surgery, but reversed to nonsignificant changes after 1 year (Table 1). Speech improvement was also ephemeral, being detected only for the first 6 months after implant. On-period freezing of gait showed a stable improvement during the 5 years of follow-up.

The daily time spent off, the severity of off-periods and the severity of off-period dystonia were markedly reduced in all the patients during the 5 years of observation. Dyskinesias (particularly in the on state) were improved in all the patients after implant; 14 patients (70\%) did not have any dyskinesias at the 5-year visit, whereas the remaining had only slight, nonpainful and nondisabling dyskinesias. The improvement of motor fluctuations was maintained along the 5 years.

At the time of implant, the patients were taking the following antiparkinsonian medication: levodopa (all 20 patients), pergolide (12 patients), ropinirole (4 patients), apomorphine (subcutaneous infusion in 4 patients), and bromocriptine (1 patient). Seven patients were also on clozapine.

The mean preoperative LEDD $(1457.6 \pm 785.6)$ was greatly reduced during the first 6 months after implant and stabilized at values around $38 \%$ of the preimplant dose by 5 years (Fig. 1). Two patients were without 


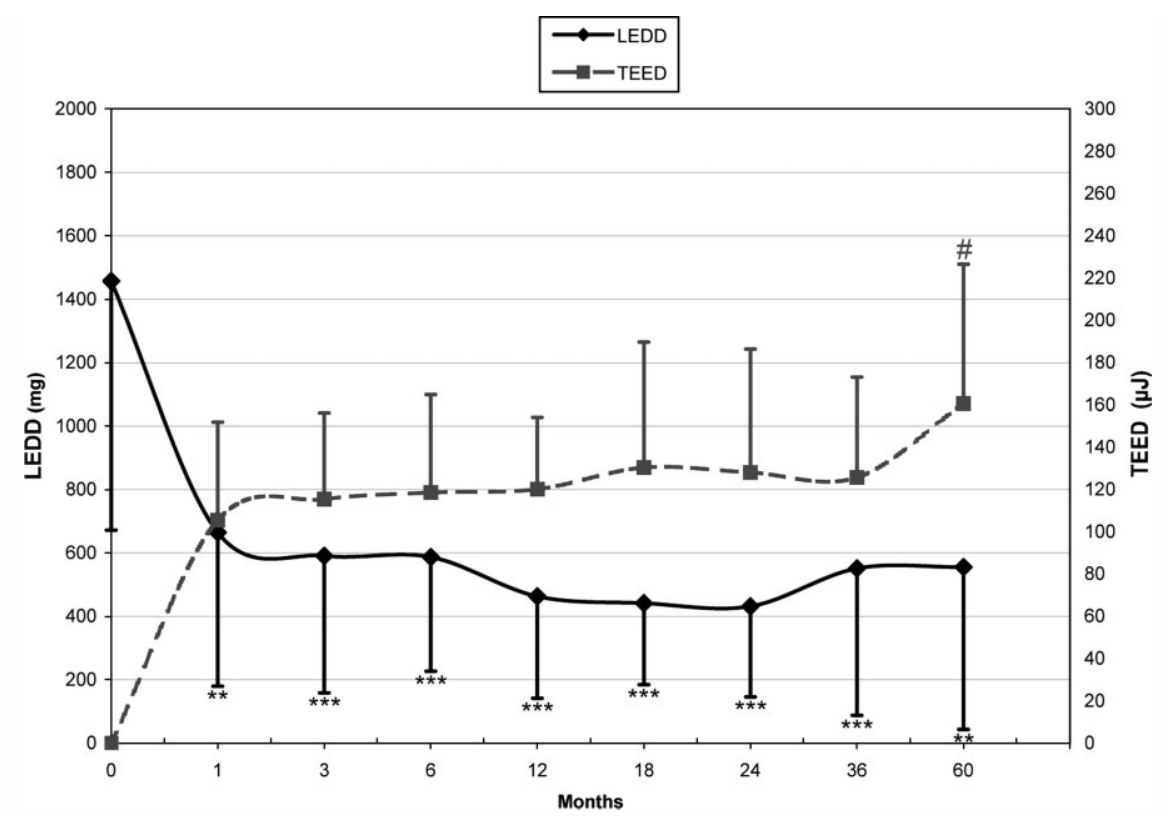

FIG. 1. Trade-off between levodopa equivalent daily dosage (LEDD) and total electrical energy delivered (TEED) during the 5-year follow-up $(\mathrm{n}=20$, mean $\pm \mathrm{SD}) .{ }^{*} P<0.005, * * * P<0.0005$ compared with baseline values; $\# P<0.05$ compared with 3-month values.

dopaminergic medication 5 years after implant, three patients took only agonists and three were only on controlled release levodopa preparations. No patient received apomorphine or clozapine.

Stimulation amplitude was progressively increased during the postoperative period, from 1 month to 5 years, to keep the best control on parkinsonian signs; pulse width, frequency, and electrode impedance remained stable. TEED did not vary until the third year and was increased afterwards (Fig. 1).

There were no significant variations in UPDRS daily living activities and in S\&E functional scale in the best postoperative motor condition compared with the best preimplant state (Table 1). During the 5 years of observation, five patients could recover a full activity in their work, whereas two retired. There were no changes in the patients' family setting (including separations or divorces).

When the patients were evaluated without medication and with stimulation turned off 5 years after implant, there was recurrence of rest tremor in 13 . Tremor intensity was on average $31 \%$ milder than that observed before implant without medication $(P<$ 0.005). There were no differences in the total UPDRS motor score compared with baseline without medication; speech, gait, and postural stability had nonsignificant variations. In some patients, off-period dystonia appeared in the face or limbs in a matter of few minutes following discontinuation of stimulation. In some of them, off-period dystonia was more disabling than before implant in the condition without medication.

Adverse events were reported in a minority of the patients (Table 2). Hypophonia occurred in three patients and was always associated with dysarthria. Two patients required botulinum toxin treatment for bilateral eyelid opening apraxia during the first year after surgery, but not later on. One additional patient had unilateral blepharospasm that was clearly related to stimulation and resolved with botulinum toxin treatment. All the patients gained weight after surgery. Unexplained switching-off occurred in one Itrel II and one Kinetra: these events required emergency management of two patients.

\section{DISCUSSION}

The long-term effect of STN stimulation on PD patients consisted in a sustained and marked improvement of the UPDRS motor score, with reduction of severity and duration of dyskinesias and of off-periods compared with the preimplant state. LEDD was reduced by $919.3 \mathrm{mg}(61 \%)$ and TEED was $160.7 \mu \mathrm{J}$, 5 years after STN implant. The dyskinesia score 
TABLE 2. Adverse events observed in the patient series

\begin{tabular}{ll}
\hline & $\mathrm{N}$ \\
\hline Number of patients & 20 \\
Transient & \\
Increased sexuality & $2(10 \%)$ \\
Manic psychosis & $3(15 \%)$ \\
Apathy & $2(10 \%)$ \\
Seizure (responsive to antiepileptic drug) & $1(5 \%)$ \\
Persistent & $3(15 \%)$ \\
Hypophonia & $3(15 \%)$ \\
Hypophonia and dysarthria & $1(5 \%)$ \\
Hypophonia, dysarthria, oral district & \\
dystonia, dysphagia & $2(10 \%)$ \\
Eyelid opening apraxia & $4(20 \%)$ \\
Apathy & $4(20 \%)$ \\
Limb dystonia & $1(5 \%)$ \\
Buccinators spasm & $4(20 \%)$ \\
Troublesome weight gain & $1(5 \%)$ \\
Stimulation-induced & $2(10 \%)$ \\
Hypophonia & $1(5 \%)$ \\
Limb dystonia & $2(10 \%)$ \\
Unilateral blepharospasm & \\
Monolateral buccinators spasm & $2(10 \%)$ \\
Device-related & $1(5 \%)$ \\
Unexplained switching-off & \\
Cable dehiscence due to infection & $3(15 \%)$ \\
Unrelated to procedure or stimulation & $2(10 \%)$ \\
Severe spinal arthrosis & $1(5 \%)$ \\
Transitory ischemic attack & $1(5 \%)$ \\
Cardiac arrhythmia & $1(5 \%)$ \\
Cardiac ischemia & $1(5 \%)$ \\
Cardiac decompensation & \\
Persistent glottal edema & \\
\hline &
\end{tabular}

The table reports the number of patients with adverse events and the percent incidence.

improved by $80 \%$, more than the motor score, which improved by $54.2 \%$. TEED was stable until the third year and then increased by $21.8 \%$ at 5 years; by contrast, LEDD was reduced during the first 6 months after implant without any further variation. This indicates that, under these experimental circumstances, progressively more energy was required to maintain control of parkinsonian symptoms over time. Previous long-term observations, which have not considered the TEED as an outcome measure, reported voltage increase after STN implants. 5,10,11

Several factors may have contributed to TEED increase. First, disease progression over 5 years may have been compensated by an increase in stimulation, given the stability of dopaminergic treatment. A shortterm PET study indeed showed that disease progression takes place after STN implants. ${ }^{12}$ We always evaluated the patients' motor condition without medication or stimulation, to get information on the underlying natural disease progression, and saw no changes 5 years after implant compared with baseline values. This is consistent with other long-term observations ${ }^{11}$ and likely indicates that treatment withdrawal did not last enough to allow assessing the underlying disease severity. In keeping with this, parkinsonian tremor did not recur completely in our patients. Discontinuance of stimulation over $30 \mathrm{~min}$ caused a worsening of all motor symptoms that most patients considered unacceptable, consistently with the report that sudden switch-off of STN stimulation may require emergency management in the patients who remain devoid of treatment. ${ }^{13}$ A second factor possibly contributing to TEED increase may have been the development of glial scar tissue around the stimulating electrodes, causing partial electrical insulation starting from the third year after implant. Although theoretically possible, this remains an unlikely explanation, as it has been demonstrated that tissue reaction around the electrode tip is usually minimal. ${ }^{14}$

It has been proposed that the reduction of dyskinesias can be a direct aftermath of reduced dopaminergic medication $^{15}$ leading to a down-regulation of dopamine receptors. In keeping with this, the dyskinesia score was reduced almost to 0 at 6 months and did not diverge afterwards. Still, the observation of a disproportionate improvement of motor and dyskinesia scores is also consistent with the possibility that prolonged continuous STN stimulation may also exert an indirect antidyskinetic action, probably mediated by plastic changes of corticostriatal terminals that counteract the effects of dopaminergic priming. ${ }^{16,17}$ Another possible mechanism may be related to a specific antidyskinetic effect of STN stimulation, similar to that of pallidal stimulation, due to an effect on the ansa lenticularis that runs ventrally to the STN and contains pallidal outflow to the thalamus. ${ }^{18,19}$ Whatever the mechanism, STN stimulation is particularly indicated in PD patients with prominent dyskinesias.

Functional measures showed no change after implant. The S\&E functional scale improved transiently for the first year after implant and UPDRS activities of daily living had nonsignificant changes, despite a permanent improvement in motor function. This suggests that, after STN implant, the patients encountered difficulties other than motor impairment that impaired their daily initiatives. It is quite possible that difficulties in re-adaptation of personal, familial, and socioprofessional status following motor improvement may have influenced the S\&E scale. This discrepancy between motor and functional outcome supports the view that STN implants could be performed earlier in the disease course to achieve benefit on a broader number of outcome measures. ${ }^{20}$ 
Analysis of individual items of the UPDRS motor score showed that some motor features improved more than others. Axial symptoms (namely, postural stability and speech) improved during the first year of observation, but their response to stimulation progressively deteriorated afterward. These symptoms are known not to respond to an increase of oral medication ${ }^{21,22}$ and in the present series TEED increase was also ineffective on these levodopa-resistant symptoms.

The results of this long-term observation raise the question whether motor improvement after STN stimulation is comparable with that produced by dopaminergic medication. Medication-insensitive worsening of axial symptoms has been attributed to the extension of neural degeneration to nondopaminergic areas. ${ }^{21}$ Axial symptoms are resistant to STN stimulation as much as to levodopa or dopamine agonists. Short-term improvement of postural stability has been correlated to an effect on the nondopaminergic connection running from the STN to the pedunculopontine nucleus. ${ }^{23,24}$ We have observed that improvement of postural instability reverses after 1 year, indicating that transient diaschisis, rather than permanent changes, can occur via this direct connection. ${ }^{25}$

We also show here that mild, medication dependent, hallucinations can benefit from STN stimulation. All the seven patients who were on clozapine before implant had no postoperative recurrence of hallucinations. It is likely that the management of mild psychotic symptoms was directly caused by the reduction of dopaminergic medication after implant. Overall, these data indicate that chronic STN stimulation is efficacious in controlling levodopa-responsive parkinsonian symptoms and allows maintaining a long-lasting reduction of dopaminergic treatment for 5 years given a progressive TEED increase. Autonomy and daily living activities are maintained, but not improved.

Acknowledgments: The study was partly funded by the Italian Ministry of University and Research (National Interest Project number 2001062543 to A.A.). The authors wish to thank Prof. Lucio Braicovich, Dipartimento di Fiscia, Politecnico diMilano, for his aid, TEED formula interpretation and usage. The contributions of the authors are as follows: L. Romito--Research project and statistical analysis: execution, manuscript: writing of the first draft; F. Contarino-Research project: execution; N. VanacoreStatistical analysis: design, review and critique; A.R. Bentivoglio-Research project: organization; M. Scerrati-Research project: execution; A. Albanese-Research project: conception, manuscript: review and critique.

\section{REFERENCES}

1. Limousin P, Pollak P, Benazzouz A, et al. Effect of parkinsonian signs and symptoms of bilateral subthalamic nucleus stimulation. Lancet 1995;345:91-95.
2. Moro E, Scerrati M, Romito LM, Roselli R, Tonali P, Albanese A. Chronic subthalamic nucleus stimulation reduces medication requirements in Parkinson's disease. Neurology 1999;53:8590 .

3. Kleiner-Fisman G, Herzog J, Fisman DN, et al. Subthalamic nucleus deep brain stimulation: summary and meta-analysis of outcomes. Mov Disord 2006;21(Suppl 14):S290-S304.

4. Liang GS, Chou KL, Baltuch GH, et al. Long-term outcomes of bilateral subthalamic nucleus stimulation in patients with advanced Parkinson's disease. Stereotact Funct Neurosurg 2006; 84:221-227.

5. Wider C, Pollo C, Bloch J, Burkhard PR, Vingerhoets FJ. Longterm outcome of 50 consecutive Parkinson's disease patients treated with subthalamic deep brain stimulation. Parkinsonism Relat Disord 2008;14:114-119.

6. Hughes AJ, Daniel SE, Kilford L, Lees AJ. Accuracy of clinical diagnosis of idiopathic Parkinson's disease: a clinico-pathological study of 100 cases. J Neurol Neurosurg Psychiatry 1992;55:181184.

7. Defer GL, Widner H, Marie RM, Remy P, Levivier M. Core assessment program for surgical interventional therapies in Parkinson's disease (CAPSIT-PD). Mov Disord 1999;14:572584.

8. Koss AM, Alterman RL, Tagliati M, Shils JL. Calculating total electrical energy delivered by deep brain stimulation systems. Ann Neurol 2005;58:168.

9. Romito LM, Scerrati M, Contarino MF, Bentivoglio AR, Tonali P, Albanese A. Long-term follow up of subthalamic nucleus stimulation in Parkinson's disease. Neurology 2002;58:15461550 .

10. Krack P, Batir A, van Blercom N, et al. Five-year followup of bilateral stimulation of the subthalamic nucleus in advanced Parkinson's disease. N Engl J Med 2003;349:19251934.

11. Schupbach WM, Chastan N, Welter ML, et al. Stimulation of the subthalamic nucleus in Parkinson's disease: a 5 year follow up. J Neurol Neurosurg Psychiatry 2005;76:1640-1644.

12. Hilker R, Portman AT, Voges J, et al. Disease progression continues in patients with advanced Parkinson's disease and effective subthalamic nucleus stimulation. J Neurol Neurosurg Psychiatry $2005 ; 76: 1217-1221$.

13. Hariz MI, Johansson F. Hardware failure in parkinsonian patients with chronic subthalamic nucleus stimulation is a medical emergency. Mov Disord 2001;16:166-168.

14. McClelland S, Vonsattel JP, Garcia RE, et al. Relationship of clinical efficacy to postmortem-determined anatomic subthalamic stimulation in Parkinson syndrome. Clin Neuropathol 2007;26: 267-275.

15. Benabid AL, Benazzouz A, Limousin P, et al. Dyskinesias and the subthalamic nucleus. Ann Neurol 2000;47(Suppl 1):S189S192.

16. Bejjani BP, Arnulf I, Demeret S, et al. Levodopa-induced dyskinesias in Parkinson's disease: is sensitization reversible? Ann Neurol 2000;47:655-658.

17. Oueslati A, Sgambato-Faure V, Melon C, et al. High-frequency stimulation of the subthalamic nucleus potentiates L-DOPAinduced neurochemical changes in the striatum in a rat model of Parkinson's disease. J Neurosci 2007;27:2377-2386.

18. Katayama Y, Oshima H, Kano T, Kobayashi K, Fukaya C, Yamamoto T. Direct effect of subthalamic nucleus stimulation on levodopa-induced peak-dose dyskinesia in patients with Parkinson's disease. Stereotact Funct Neurosurg 2006;84:176179.

19. Herzog J, Pinsker M, Wasner M, et al. Stimulation of subthalamic fibre tracts reduces dyskinesias in STN-DBS. Mov Disord 2007;22:679-684.

20. Schupbach WM, Maltete D, Houeto JL, et al. Neurosurgery at an earlier stage of Parkinson disease: a randomized, controlled trial. Neurology 2007;68:267-271. 
21. Bonnet AM, Loria Y, Saint-Hilaire MH, Lhermitte F, Agid Y. Does long-term aggravation of Parkinson's disease result from nondopaminergic lesions? Neurology 1987;37:15391542 .

22. Clissold BG, McColl CD, Reardon KR, Shiff M, Kempster PA. Longitudinal study of the motor response to levodopa in Parkinson's disease. Mov Disord 2006;21:2116-2121.

23. Breit S, Bouali-Benazzouz R, Benabid AL, Benazzouz A. Unilateral lesion of the nigrostriatal pathway induces an increase of neuronal activity of the pedunculopontine nucleus, which is reversed by the lesion of the subthalamic nucleus in the rat. Eur J Neurosci 2001;14:1833-1842.

24. Florio T, Scarnati E, Confalone G, et al. High-frequency stimulation of the subthalamic nucleus modulates the activity of pedunculopontine neurons through direct activation of excitatory fibres as well as through indirect activation of inhibitory pallidal fibres in the rat. Eur J Neurosci 2007;25:1174-1186.

25. Stefani A, Lozano AM, Peppe A, et al. Bilateral deep brain stimulation of the pedunculopontine and subthalamic nuclei in severe Parkinson's disease. Brain 2007;130:1596-1607. 